\title{
Molecular markers characterization determining cell fate specification in an adult neurogenesis model of Alzheimer's disease
}

\section{Solving neurogenesis in an AD model}

Idoia Blanco-Luquin ${ }^{1}$ Ph.D.* , Juan Cabello ${ }^{2}$ Ph.D., Amaya Urdánoz-Casado ${ }^{1}$ M.Sc., Blanca Acha $^{1}$, Eva Ma Gómez-Orte ${ }^{2}$ Ph.D., Miren Roldan ${ }^{1}$, Diego R. Pérez-Rodríguez ${ }^{3}$ M.D., Maite Mendioroz ${ }^{1,4}$ M.D., Ph.D.

1. Neuroepigenetics Laboratory-Navarrabiomed, Complejo Hospitalario de Navarra, Universidad Pública de Navarra (UPNA), IdiSNA (Navarra Institute for Health Research), Pamplona, Navarra, 31008, Spain

2. CIBIR (Center for Biomedical Research of La Rioja), Logroño, La Rioja, 26006, Spain

3. Neurophysiology Department. Complejo Hospitalario de Navarra- IdiSNA (Navarra Institute for Health Research), Pamplona, Navarra, 31008, Spain

4. Department of Neurology, Complejo Hospitalario de Navarra- IdiSNA (Navarra Institute for Health Research), Pamplona, Navarra, 31008, Spain

*Corresponding author: Idoia Blanco-Luquin

Email: idoia.blanco.luquin@gmail.com 
bioRxiv preprint doi: https://doi.org/10.1101/2020.08.06.239111; this version posted August 6, 2020. The copyright holder for this preprint (which was not certified by peer review) is the author/funder. All rights reserved. No reuse allowed without permission.

\section{2}

\section{SUMMARY STATEMENT}

Transcriptional profile of a number of genes recapitulating particular stages of Adult hippocampal neurogenesis in the context of Alzheimer's disease 


\section{ABSTRACT}

Adult hippocampal neurogenesis (AHN) study is still a challenge. In addition to methodological difficulties is the controversy of results derived of human or animal system approaches. In view of the proven link between AHN and learning and memory impairment, we generated a straightforward in vitro model to recapitulate adult neurogenesis in the context of Alzheimer's disease (AD).

Neural progenitor cells (NPCS) monolayer culture was differentiated for a period of 29 days and $A \beta$ peptide 1-42 was administered once a week. mRNA expression of NEUROD1, NCAM1, TUBB3, RBFOX3, CALB1 and GFAP genes was determined by RT-qPCR.

Phenotypic changes were observed during directed differentiation. Except for GFAP and CALB1, these changes correlated with altered expression profile of all genes since 9 days. Only TUBB3 expression remained constant while NEUROD1, NCAM1 and RBFOX3 expression increased over time. Moreover, A $\beta$ treated NPCs showed transient decreases of mRNA expression for NCAM1, TUBB3 and RBFOX3 genes at 9 or 19 days.

Our in vitro human NPCS model is framed within the multistep process of AHN in the SGZ of the DG. Remarkably, its transcriptional assessment might reflect alterations detected in $A D$ human patients, deepening our understanding of the disorder and possibly of its pathogenesis.

Keywords: Adult hippocampal neurogenesis; NPCs; Alzheimer's disease; A $\beta$ peptide; Gene expression. 
1

2

3

4

5

6

7

8

9

\section{INTRODUCTION}

Adult neurogenesis is the process of forming functional neurons de novo. In an adult mammalian brain, neurogenesis occurs predominantly in specific brain niches: the subgranular zone (SGZ) of the dentate gyrus (DG) of the hippocampus and the subventricular zone (SVZ) lining the lateral ventricles (Hollands, Bartolotti and Lazarov, 2016; Hsieh, 2012). During the process of adult hippocampal neurogenesis (AHN), neural stem cells (NSCs) selfrenew and differentiate, giving rise to transient amplifying progenitors (TAPs), neuroblasts, and eventually mature neurons, astrocytes, and oligodendrocytes. AHN regulators can be divided into intrinsic or extrinsic factors, that is, transcription factors (TFs) synthesized by the developing neural precursors and neurons, and growth factors and neurotrophins secreted from the surrounding niche, respectively (Covic, Karaca and Lie, 2010).

Understanding of human nervous system biology relies on the availability of human NSCS and their differentiated derivatives. A great deal of effort has been put into the generation of neural progenitor cells (NPCS) and neurons in vitro, mostly by differentiation of pluripotent stem cells (PSCs) and more recently by genetically reprogramming somatic cells to an embryonic stem (ES) cell-like state (induced pluripotent stem cells, iPSCS) (Yu, Marchetto and Gage, 2014). NPCs constitute an intermediate stage between pluripotent immature cells, such as ES cells and mature differentiated neural cells.

AHN clearly emerges as a robust phenomenon during both physiological and pathological aging in humans (Mu and Gage, 2011). In addition, the fact that it is implicated in normal functionality of hippocampal circuits, demonstrates an important link between adult neurogenesis and cognitive processes (Baglietto-Vargas et al., 2017). As a consequence, impaired neurogenesis may negatively impact the survival of adult-born neurons and contribute to learning and memory failure, such as the associated with aging and disorders such as Alzheimer's Disease (AD) (Mu and Gage, 2011; Coronel et al., 2018; Li, Bao and Wang, 2016).

$A D$ is the most common neurodegenerative disorder, characterized by progressive memory loss and cognitive decline caused by widespread neurons loss and synaptic connections in 
the cortex, hippocampus, amygdala and basal forebrain, and by a gradually significant loss of brain mass. Amyloid precursor protein (APP) plays a key role in normal brain development by influencing NSC proliferation, cell fate specification and neuronal maturation (Coronel et al., 2018). However, its derivative Amyloid $\beta$ (AB) peptide, a cleavage product of APP enzymatic processing, is the major component of the amyloid plaques, one of the hallmark pathologies found in brains of late-onset sporadic $A D$ patients. Monomeric $A B$ can self-aggregate to form oligomers, protofibrils, and amyloid fibrils which deposit as these amyloid plaques. Despite $A \beta$ impact on neurogenesis is still controversial, $A \beta$ plaques can cause severe damage to neurons and astrocytes, resulting in a gradual loss of neurons behind AD symptoms ( $L i$, Bao and Wang, 2016).

Interestingly, noteworthy alterations in AHN have been detected at early stages of the disease, even before the onset of hallmark lesions or neuronal loss (Mu and Gage, 2011; Moreno-Jimenez et al., 2019). Hence, a better understanding of AHN impairment observed at initial and later stages of $A D$ by noninvasive methods might reveal insights into the pathogenesis of AD. What is more, restoration of normal levels of AHN would provide a potential therapeutic strategy to delay or halt AD-linked cognitive decline (Mu and Gage, 2011; Moreno-Jimenez et al., 2019).

Here, we propose an intuitive in vitro approach to follow a stepwise lineage progression, as occurs during in vivo neurogenesis, by using human NPCs derived from an iPSC line as the starting source material. In order to find out whether human NPCs differentiation into mature neurons of any functional classification is disrupted in the AD microenvironment, we generated an in vitro model triggered by prolonged exposure towards nanomolar concentrations of $A \beta$ peptide and characterized it by assessing a set of neurogenesis markers.

\section{MATERIALS AND METHODS}

\section{NPCs culture, neuronal differentiation and $A \beta$ peptide administration}

Neural Progenitor Cells (NPCS) Derived from XCL1 DCXpGFP (ATCC ${ }^{\circledast}$ ACS5005 ${ }^{\mathrm{TM}}$ ) were cultured following manufacturer recommendations. Briefly, $0.30 \times 10^{6} \mathrm{NPCs}$ were seeded onto a CellMatrix Basement Membrane Gel (ATCC ${ }^{\circledR}$ ACS3035 $^{\mathrm{TM}}$ ) coated 12-well plate and 
incubated in NPC expansion medium: complete growth medium including DMEM/F-12 (Gibco, Fisher Scientific) supplemented with the Growth Kit for Neural Progenitor Cell Expansion (ATCC ${ }^{\circledast}$ ACS3003) and then maintained in a humidified incubator $\left(5 \% \mathrm{CO} 2,37^{\circ} \mathrm{C}\right)$.

Neuronal differentiation experiments were carried out for 9,19 and 29 days by plating NPCs at a seeding density of 80,000 viable cells/cm2 in 6 -well coated culture plates. First, NPCs were incubated in expansion medium, which is referred to as day 0 in this study. Day post seeding, half-medium changes with differentiation medium were performed every 2-3 days throughout the duration of the culture period. Complete Differentiation Medium consisted of serum-free neuronal basal BrainPhys ${ }^{\mathrm{TM}}$ Neuronal Medium formulated to improve the electrophysiological and synaptic properties of neurons (Satir et al., 2020), NeuroCult ${ }^{\text {TM }}$ SM1 Neuronal Supplement (1:50), N2 Supplement-A (1:100), Recombinant Human Brain-Derived Neurotrophic Factor (BDNF, $20 \mathrm{ng} / \mathrm{mL}$ ), Recombinant Human Glial-Derived Neurotrophic Factor (GDNF, $20 \mathrm{ng} / \mathrm{mL}$ ), Dibutyryl-cAMP (1 mM) and ascorbic acid (200 nM) (STEMCELL Technologies, Vancouver, BC, Canada). Half fresh medium containing Amyloid $\beta$ Protein Fragment 1-42 (50 nM; Sigma-Aldrich, St. Louis, MO, USA) or DMSO (Sigma-Aldrich) as vehicle was added once a week.

NPCs were harvested at 0 or 9,19 and 29 days of differentiation for both conditions, by detaching with Accutase (Innovative Cell Technologies, San Diego, CA), washed with Dulbecco's phosphate-buffered saline (DPBS, Sigma-Aldrich), centrifuged at 13000 r.p.m. and frozen at $-80 \stackrel{\circ}{\circ}$. All experiments were performed in triplicate.

\section{Neurogenesis markers mRNA expression analysis by RT-qPCR}

Total RNA was extracted from frozen cell pellets of basal NPCs and control or A $\beta$ treated NPCs incubated in differentiation media for 9, 19 or 29 days using RNeasy Mini kit (QIAGEN, Redwood City, CA, USA), following manufacturer's instructions. Genomic DNA was digested with DNase I (RNase-Free DNase Set, Qiagen). Concentration and purity of RNA were both evaluated with NanoDrop spectrophotometer. Only RNA samples showing a minimum quality index $(260 \mathrm{~nm} / 280 \mathrm{~nm}$ absorbance ratios between 1.8 and 2.2 and $260 \mathrm{~nm} / 230 \mathrm{~nm}$ absorbance ratios higher than 1.8) were included in the study. Complementary DNA (CDNA) was reverse transcribed from 1000 ng total RNA with SuperScript ${ }^{\circledR}$ III First-Strand Synthesis 
87 Reverse Transcriptase (Invitrogen, Carlsbad, CA, USA) after priming with oligo-d ( $T$ ) and random primers. RT-qPCR reactions were performed in duplicate with Power SYBR Green PCR Master Mix (Invitrogen, Carlsbad, CA, USA) in a QuantStudio 12K Flex Real-Time PCR System (Applied Biosystems, Foster City, CA, USA). Sequences of primer pair were designed using Real Time PCR tool (IDT, Coralville, IA, USA) and are listed in Supplementary Table S1. Relative expression mRNA levels of lineage specific genes in a particular sample were calculated as previously described (Livak and Schmittgen, 2001) and geometric mean of ACTB and GAPDH genes was used as the reference to normalize expression values.

\section{Immunofluorescence Staining}

NPCS were seeded on Nunc ${ }^{\text {TM }}$ Lab-Tek $^{\text {TM }}$ II chamber slides (Thermo Fisher Scientific, Waltham, MA, USA), coated with CellMatrix Basement Membrane Gel. Cells were either left untreated, or treated with Amyloid $\beta$ Protein Fragment 1-42 (50 $\mathrm{nM})$, in differentiation media as described above, and after 9,19 or 29 days of incubation, fixated in $4 \%$ formalin (OPPAC, Spain) for $15 \mathrm{~min}$. Cells were permeabilized using 0.5\% TWEEN ${ }^{\circledR} 20$ (Sigma-Aldrich) in DPBS, then blocked with $10 \%$ fetal bovine serum (Sigma-Aldrich) containing 0.5\% Tween in DPBS 30 min at room temperature. Rabbit monoclonal anti-NeuN [EPR12763] (Cat\# ab177487, RRID:AB_2532109; 1:300), anti-GFAP [EP672Y] (Cat\# ab33922, RRID:AB_732571; 1:300), antiSynaptophysin [YE269] (Cat\# ab32127, RRID:AB_2286949; 1:200) and anti-Ki67 [SP6] (Cat\# ab16667, RRID:AB_302459; 1:500) primary antibodies (Abcam, Cambridge, UK) diluted in blocking buffer were added, and incubated overnight at $4 \stackrel{\circ}{ } \mathrm{C}$. After three washing steps, Alexa Fluor $^{\circledR} 647$ donkey anti-rabbit secondary antibody (Abcam Cat\# ab150075, RRID:AB_2752244; 1:500) was added, and incubated for 30 minutes at room temperature in the dark. Following three washing steps, the slides were mounted with ProLong ${ }^{\text {TM }}$ Gold Antifade Mountant with DAPI (Molecular Probes, OR, USA). Immunofluorescence images were acquired using a Cytation 5 Cell Imaging Multi-Mode Reader and analyzed with Gen5 ${ }^{\mathrm{TM}}$ software (BioTek, Winooski, VT, USA).

\section{Statistical data analysis}

Statistical analysis was performed with SPSS 21.0 (IBM, Inc., USA) and GraphPad Prism version 6.00 for Windows (GraphPad Software, La Jolla, CA, USA). First, we checked that all 
116 continuous variables showed a normal distribution, as per one-sample Shapiro-Wilk test.

117 Data represents the mean \pm standard error of the mean (SEM). Significance level was set at

$118 p$-value $<0.05$. Statistical significance for neuronal lineage specific genes mRNA levels across

119 different time points was assessed by one-way analysis of variance (ANOVA) followed by 120 post-hoc analyses Tukey's honestly significant difference (HSD) and Dunnett's multiple 121 comparisons tests. In cases where Levene test did not show homogeneity of variance, 122 Welch's ANOVA followed by Dunnett's T3 was conducted.

Paired t-test was used to analyze differences in the expression levels of the studied genes mRNA between $A \beta$ treated and control group at each time point. GraphPad Prism version 6.00 for Windows was used to draw graphs.

RESULTS

To determine whether neural differentiation was effectively induced, we observed the morphological modifications of the cells over time. As shown in Figure 1, NPCs exposure to differentiation medium caused an increase in the number and length of the neuritic extensions, which can even connect with the extensions of neighbor cells, compared with basal cells grown in proliferation medium at zero time. These changes in cell morphology are typical of cells undergoing differentiation (Compagnucci et al., 2016), were noticed since the first time point at 9 days, and became more evident as time went on in response to directed neurogenesis. Furthermore, total cell number in NPCs cultures remained steady, due to an absence of proliferation, stated by unchanged Ki 67 protein marker expression

137 (Supplementary Figure S1), which correlated with a gradually cell differentiation boost. As a matter of fact, immunofluorescence (IF) staining proved NeuN (neuronal nuclei), GFAP (glial fibrillary acidic protein) and Synaptophysin protein expression, which mark neurons, glial cells and synaptic vesicles respectively, across the NPCs culture (Figure 2).

To confirm the above observations, we explored if gene expression profiles of different transcription factors and molecular markers changed in our in vitro model across consecutive stages of driven neuronal differentiation. For that purpose, we measured mRNA expression 
144 levels of Neuronal Differentiation 1 (NEUROD1), Neural Cell Adhesion Molecule 1 (NCAM1), 145 Tubulin Beta 3 Class III (TUBB3), RNA Binding Fox-1 Homolog 3 (RBFOX3), Calbindin 1 (CALB1) and GFAP genes by RT-qPCR, regardless of the treatment with $A \beta$ (Figure 3 ).

NEUROD1 mRNA expression levels of NPCs cultured in differentiation medium increased from 9 to 19 days [mean difference \pm standard error (SE), 0.03 \pm 0.009 ; $p$-value $=0.048$ ]. Moreover, we quantified a statistically significant elevation of mRNA expression for this basic helix-loop-helix (bHLH) TF at $19(0.047 \pm 0.008 ; p$-value<0.0001) and 29 days $(0.027 \pm 0.008 ; p$ value $=0.009$ ) compared with basal cells.

In our in vitro model, NCAM1 mRNA expression overlapped that of NEUROD1 gene, although we found a statistically significant raise since the addition of differentiation medium to the cell culture (ANOVA, $p$-value $<0.001)$, which is more pronounced at 19 days $(5.956 \pm 0.468) ; p$ value<0.0001). Our results also displayed statistically significant differences between 9 to 19 days (3.122 \pm 0.599 ; $p$-value<0.001), 9 to 29 days (2.554 $\pm 0.599 ; p$-value=0.002) and between basal cells and any of the other time points: from 0 to 9 days $(2.834 \pm 0.468 ; p$-value $<0.0001)$ and from 0 to 29 days $(5.389 \pm 0.468 ; p$-value $<0.0001)$.

Once the proliferation medium was exchanged by differentiation medium, NPCs began to express TUBB3 mRNA, a gene marker that plays a critical role in proper axon guidance and maintenance. This increase remained constant over time comparing with basal cells (6.288 \pm 1.002 from 0 to 9 days, $7.351 \pm 1.002$ from 0 to 19 days and $7.412 \pm 1.002$ from 0 to 29 days; with a p-value<0.0001 for all of them). However, no changes were observed between first, second and third time points.

RBFOX3 encodes the NeuN antigen that has been widely used as a marker for post-mitotic neurons. In our study, RBFOX3 mRNA expression grows in time proving the successful achievement of progenitor-to-neuron differentiation. This progressive increase showed statistically significant differences in mRNA expression between 0 and 9 days $(0.0105 \pm 0.001$; $p$-value $=0.001), 9$ and 19 days $(0.03 \pm 0.005 ; p$-value $=0.007)$ or 9 and 29 days $(0.057 \pm 0.014 ; p$ value=0.047). Besides, all other differences between any time point with respect to basal

171 cells were also found statistically significant: from 0 to 19 days $(0.04 \pm 0.005 ; p$-value=0.002) 172 and from 0 to 29 days $(0.067 \pm 0.014 ; p$-value $=0.024)$. 
173 In relation to CALB1 mRNA expression, and given that this gene encodes a protein expressed

174 in mature granule cells, we did not detect any significant change.

175

176

177

178

179

180

181

GFAP mRNA expression observed coincided in time with that of the neuron lineage-specific gene marker $R B F O X 3$, and it was not present in the proliferative stage, at time zero. On the contrary, we noticed a slow raise of GFAP expression at 19 days, which was statistically significant compared with basal cells $(0.004 \pm 0.001$; p-value $=0.004)$ and 9 days of differentiation $(0.003 \pm 0.001 ; p$-value $=0.009)$. This pointed to the existence of NPCs derived astrocytes in the culture.

None of the studied genes showed significant differences of mRNA expression between 19 days and the end time point.

\section{Effect of $A \beta$ peptide addition on cultured NPCs during neurogenesis stages}

In order to mimic the AD context, we exposed NPCs to $A \beta$ peptide once a week throughout the incubation period with differentiation medium. Thus, we wanted to assess whether expression levels of the genes chosen to characterize the stage of neurogenesis in culture were altered due to $A \beta$ peptide addition.

We found transient treatment-specific differences in mRNA expression for some of the studied lineage-specific genes (Figure 4). A $\beta$ peptide reduced NCAM1 expression [mean of differences \pm standard error of the mean (SEM), 1.572 \pm 0.343 ]; $p$-value=0.044) at 19 days and TUBB3 (1.518 \pm 0.296$)$ and RBFOX3 (0.004 \pm 0.0003$) ; p$-value $=0.008)$ expression at 9 days. Interestingly, such decreases occurred at the beginning or in between the studied time window, but these differences were no longer significant at the end time point.

\section{DISCUSSION}

Nowadays, a comprehensive overview has emerged about the stages of AHN development. This complex multistep process can be divided into four phases: a precursor cell phase, an early survival phase, a postmitotic maturation phase, and a late survival phase. Type 1 radial glia-like cells (RGLS) represent the NSC population and can give rise to TAPs (type 2 cells), with first glial (type $2 \mathrm{a}$ ) and then neuronal (type $2 \mathrm{~b}$ ) phenotype. Through a migratory 
200 neuroblast-like stage (type 3), lineage-committed cells exit the cell cycle ahead of 201 maturation into dentate granule neurons functionally integrated into the hippocampal 202 circuitry (Kempermann, Song and Gage, 2015; Zhang and Jiao, 2015). Based on cell morphology, 203 TFs expression and a set of marker proteins, distinct milestones have been established 204 (Kempermann, Song and Gage, 2015). In this study, we examined the expression dynamics of key markers to characterize a directed human NPCs differentiation model across different developmental stages (Figure 5).

During stage 1 (proliferation phase), newly generated cells express GFAP. However, we do not detect differences in GFAP expression until day 19 after the addition of differentiation medium. This leads to believe that our in vitro NPCs culture window starts after the proliferative phase, since type-2 cells (differentiation phase) lose the GFAP marker (Zhang and Jiao, 2015). Then, knowing that, in contrast to their in vivo counterparts in SGZ of the brain, the in vitro expanded NSCs are less neurogenic and mainly biased towards an astrocytic fate upon differentiation (Azari, 2013), GFAP expression at day 19 would correspond to a subset of astrocytes present in our NPCs culture (Pierret et al., 2010).

In stage 3 (migration phase) migrating neuroblasts display the polysialylated form of NCAM (PSA-NCAM), a marker that arrives at the late strategy of adult neurogenesis and seems to persist in young postmitotic neurons (von Bohlen Und Halbach, 2007). Accordingly, our results suggest the existence of a plateau between 19 and 29 days for NCAM1 mRNA expression. Most PSA-NCAM-positive cells express NeuroD and NeuN, but not GFAP, which argues in our favor with respect to our above mentioned findings (von Bohlen Und Halbach, 2007). bHLH TF NEUROD1, expressed in later stages of neuronal commitment, plays an essential role in the differentiation and survival of neuronal precursors. NeuroD1 deletion leads to new granule neurons depletion and their failure to integrate in the DG (Shohayeb et al., 2018). In line with this, we observed a rise of NEUROD1 expression during our culture time window, as reported by Xuan Yu et al. (Yu et al., 2014). Meanwhile, NeuroD expression can also be detected in PSA-NCAM-positive cells, precedes it (von Bohlen Und Halbach, 2007) and reaches the highest point in late stage type $2 b$ and type 3 cells (Hsieh, 2012), although we did not identify this peak in our model. On its part, once the newly generated 
230 an earlier RBFOX3 mRNA expression in our model. We found how RBFOX3 expression 231 increased until 19-29 days of differentiation, showing a similar expression profile than 232 NCAM1.

Next, cells become postmitotic entering stage 4 (axonal and dendritic targeting). Immature neurons still express PSA-NCAM and, at the same time, can also be marked by NeuN. Also involved in axon guidance and maintenance, TUBB3 arises, encoding a class III member of the beta tubulin protein family, characteristic of early postmitotic and differentiated neurons and some mitotically active neuronal precursors. This is consistent with the increase of TUBB3 mRNA detected in our model, prior to be translated to protein. TUBB3 mRNA expression persists in neurons displaying high complexity and electrophysiological properties, which show immunoreactivity for NeuN and thus represent postmitotic neurons (von Bohlen Und Halbach, 2007).

Finally, mature granule cells establish their synaptic contacts and become functionally integrated in the hippocampus in stage 5 (synaptic integration), expressing calbindin together with NeuN but not co-expressing PSA-NCAM (von Bohlen Und Halbach, 2007). Our group did not find variations in CALB1 mRNA expression within the analyzed culture time window that might be supposedly expected later in time.

Therefore, based on NPCs monolayer cultured with a medium that accelerates neuronal differentiation by enhancing synaptic activity (Satir et al., 2020), we achieved the challenge of developing a less time consuming differentiation strategy that approximates the in vivo developmental program of human hippocampal DG, which differs from that of the SVZ (Ertaylan et al., 2014), as we were able to generate neurons potentially expressing many features of the process of AHN.

In the $A D$ context, it is known that $A \beta$ peptides are generated after cleavage of APP by $\gamma$ secretase in the amyloidogenic pathway (Coronel et al., 2018). Physiological concentration of $A \beta$ peptides in the brain revealed a positive effect on neuroplasticity and learning, showing improved hippocampal long-term potentiation (LTP), while high nanomolar $A \beta$ administration resulted in impaired cognition (Lazarevic et al., 2017; Garcia-Osta and Alberini, 2009), what reflects an hormetic nature (Puzzo, Privitera and Palmeri, 2012). 
259 Because extracellular concentrations of $A \beta$ in normal brain have been estimated to low 260 picomolar levels, in our experiments, we chose a concentration of $A \beta$ peptide 1-42 in the nanomolar range (50 $\mathrm{nM}$ ) to be added once a week during 29 days of culture, a single dose determined on the average of those used by Gulisano et al. (Gulisano et al., 2018) and Malmsten et al. (Malmsten et al., 2014b)

It has been reported that oligomeric synthetic $A \beta$ peptide 1-42 decreases human NSC proliferative potential and appears to favor glial differentiation, reducing neuronal cell fates (Coronel et al., 2018), or suppresses the number of functional human ES cells-derived neurons (Wicklund et al., 2010). Instead, Bernabeu-Zornoza et al. showed that $1 \mu \mathrm{M}$ monomeric A $\beta$ peptide 1-42 promoted human NSCs proliferation by increasing the glial precursors pool, without affecting neurogenesis (Coronel et al., 2019). And, on the other hand, differentiating neurospheres exposed to fibrillar $A \beta$ decreased neuronal differentiation and induced gliogenesis (Wicklund et al., 2010; Malmsten et al., 2014a). Controversies existing in the field might be due to $A \beta$ isoforms, peptides concentration, aggregation state, administration times or type of NSCS/NPCs from different species or culture systems used in each experiment (Coronel et al., 2019).

In our case, some of the genes we analyzed showed a decrease in mRNA expression with our $A \beta$ treatment scheme. This transient effect was evident at 9 or 19 days but disappeared at 29 days. It suggests that, despite affecting genes involved in neurogenesis fate, probably before cells maturation, and causing a decrease in differentiation, nanomolar range concentration $A B$ addition is somehow counteracted in the long term. A time-dependent reversal in the effects of picomolar $A \beta$ on synaptic plasticity and memory was already seen by Koppensteiner et al., attributable to the enzyme neprilysin, whose levels are reduced with aging and in AD patients brains (Koppensteiner et al., 2016). Strikingly, a study in which mutant APP was overexpressed to ensure $A \beta$ release exclusively by mature neurons found neither a positive nor a negative effect in AHN (Tincer et al., 2016). Hence, our simplistic model might shed light about early $A D$ neurogenesis events, before $A \beta$ deposition cannot be overcome.

A transcriptomic analysis of several human AD profiles demonstrated upregulation of neural progenitor markers expression and downregulation of later neurogenic markers, 
289

290

291

292

293

294

295

296

297

298

299

300

301

302

303

304

305

306

307

308

309

310

311

312

313

314

315

316

317

318

hypothesizing that neurogenesis is reduced in AD due to compromised maturation (Gatt et al., 2019). Interestingly, they showed downregulation of NCAM1 expression in early AD hippocampus and also of NCAM1, TUBB and RBFOX3 in late AD hippocampus, which is precisely in line with our $A \beta$ culture treatment results. And recently, Moreno-Jimenez et al. provided evidence for substantial impairment maturation underlying $A D$ progression. Importantly, they identified a decline in doublecortin-expressing cells that co-expressed PSANCAM in the DG starting at Braak stage III, followed by a reduction in the expression of NeuN and BlII-tubulin, among others, at some of the subsequent stages of the disease (Moreno-Jimenez et al., 2019).

AHN confers a unique mode of plasticity to the mature mammalian brain. Research about it requires non-invasive monitoring to understand its life-long impact (Bond, Ming and Song, 2015). Easier than manipulating NSCS, in part because of the time-saving, our NPCS model facilitates to study gene expression levels from an in vitro cell culture platform that can generate multiple neuronal and glial types in a human genetic context (Efthymiou et al., 2014). Moreover, this straightforward approach helps us to recapitulate $A D$ to deepen our understanding of the alterations affecting specific lineage cell types, even to observe early pathological changes, possibly associated with the prodromal phase of the disease. Nonetheless, SGZ NSCS generate dentate granule neurons and astrocytes while, once propagated in culture with the adequate concentrations of growth factors, they produce all three neural lineages (Bond, Ming and Song, 2015). On the other hand, other cell types are involved in pathogenesis, especially microglia that play a major role, together with neuroinflammation, in AD risk and progression. In consequence, it should be noted that the high potential of NSCS/NPCS could be limited by the characteristics of the in vivo niche environment.

Finally, the development of AHN monitoring methods as a biomarker of cognitive function in live individuals will be crucial to stage $A D$ progress. Moreover, to exploit the utility of transcription factor reprogramming to preserve endogenous AHN might contribute to cognitive resilience in AD (Gatt et al., 2019). However, despite exciting, the prospect of using adult NSCs therapeutically as a regenerative source needs to address neuronal integration and its impact into host mature neural circuits (Bond, Ming and Song, 2015). It will involve 
319 strategies to accomplish the NSC pool maintenance, generation of correct neuronal 320 subtypes, suppression of glial fates, and differentiation and survival of immature neurons 321 (Hsieh, 2012).

\section{CONCLUSIONS}

323 All things considered, this work provides a transcriptional profile of a number of genes 324 involved in particular stages of the AHN process to get a detailed understanding of the 325 lineage restricted fate during human neuronal differentiation. Moreover, by the 326 administration of $A \beta$ peptide 1-42 to our human NPCs culture model, we observed similar 327 findings to those obtained in human AD samples relative to NCAM1, TUBB3 and RBFOX3 328 genes expression, possibly offering an in vitro opportunity to study AHN impairment in the 329 AD context.

Supplementary Table S1. RT-qPCR primers.

\section{AUTHOR CONTRIBUTIONS}

334 IBL contributed to study concept and design, running experiments, analysis and 335 interpretation of data, figure designing and drawing and drafting/revising the manuscript for 336 content. JC contributed to design and revising manuscript for content. AU contributed to 337 figure designing and drawing and revising the manuscript for content. BA contributed to statistical analysis and revising the manuscript for content. EMGO contributed to design and revising manuscript for content. MR contributed to running experiments. DRPR contributed

340 to interpretation of data and revising manuscript for content. MM contributed to study 341 concept and design, analysis and interpretation of data, statistical analysis, study 342 supervision, drafting/revising the manuscript for content and obtaining funding. 


\section{FUNDING}

344 This research was funded by the Spanish Government through a grant from the Institute of Health Carlos III (FIS PI17/02218), jointly funded by European Regional Development Fund (ERDF), European Union, "A way of shaping Europe"; the Trans-Pyrenean Biomedical Research Network (REFBIO II-MOMENEU project) and Government of Navarra through two grants from Department of Industry of Government of Navarra ("PI058 iBEAS-Plus" and “PI055 iBEAS-Plus"). In addition, AUC received a grant "Doctorandos industriales 2018-2020" and Predoctoral grant (2019) founded by Department of Industry and Health of Government of Navarra. MM received a grant "Programa de intensificación" founded by Fundación Bancaria "la Caixa" and Fundación Caja-Navarra and "Contrato de intensificación" from the Institute of Health Carlos III (INT19/00029).

\section{ACKNOWLEDGMENTS}

We want to kindly thank Valle Coca (Navarrabiomed BrainBank, technical support), Paula Aldaz Ph.D and Imanol Arozarena Ph.D (Cancer Signalling Research Unit, Navarrabiomed, technical and scientific support), Natalia Ramirez Ph.D (Haematological Oncology Research Unit, Navarrabiomed, scientific support), Ibai Tamayo Ph.D, Arkaitz Galbete Ph.D and Julián Librero Ph.D (Methodology Unit, Navarrabiomed, technical support) for their help.

\section{COMPETING INTERESTS}

362 The authors declare that they have no conflict of interest.

ABBREVIATIONS

$A \beta$ : amyloid $\beta$; AD: Alzheimer's disease; AHN: adult hippocampal neurogenesis; ANOVA: twoway analysis of variance; APP: amyloid precursor protein; bHLH: basic helix-loop-helix; CALB1: calbindin 1; cDNA: complementary DNA; DG: dentate gyrus; ES: embryonic stem; GFAP: glial fibrillary acidic protein; HSD: honestly significant difference; IF: immunofluorescence; iPSCs: induced pluripotent stem cells; NCAM1: Neural Cell Adhesion Molecule 1; NeuN: neuronal nuclei; NEUROD1: Neuronal Differentiation 1; NPCs: neural progenitor cells; NSCs: neural stem cells; PSA-NCAM: polysialylated form of NCAM; PSCs: pluripotent stem cells; RBFOX3: RNA Binding Fox-1 Homolog 3; RGLs: radial glia-like cells; RTqPCR: real time quantitative PCR; SE: standard error; SEM: standard error of the mean; SGZ: 
subgranular zone; SVZ: subventricular zone; TAPs: transient amplifying progenitors; TFs: transcription factors; TUBB3: Tubulin Beta 3 Class III.

\section{REFERENCES}

Azari, H. (2013) 'Isolation and enrichment of defined neural cell populations from heterogeneous neural stem cell progeny', Methods Mol Biol, 1059, pp. 95-106.

Baglietto-Vargas, D., Sanchez-Mejias, E., Navarro, V., Jimenez, S., Trujillo-Estrada, L., GomezArboledas, A., Sanchez-Mico, M., Sanchez-Varo, R., Vizuete, M., Davila, J. C., Garcia-Verdugo, J. M., Vitorica, J. and Gutierrez, A. (2017) 'Dual roles of Abeta in proliferative processes in an amyloidogenic model of Alzheimer's disease', Sci Rep, 7(1), pp. 10085.

Bond, A. M., Ming, G. L. and Song, H. (2015) 'Adult Mammalian Neural Stem Cells and Neurogenesis: Five Decades Later', Cell Stem Cell, 17(4), pp. 385-95.

Compagnucci, C., Piemonte, F., Sferra, A., Piermarini, E. and Bertini, E. (2016) 'The cytoskeletal arrangements necessary to neurogenesis', Oncotarget, 7(15), pp. 19414-29.

Coronel, R., Bernabeu-Zornoza, A., Palmer, C., Muniz-Moreno, M., Zambrano, A., Cano, E. and Liste, I. (2018) 'Role of Amyloid Precursor Protein (APP) and Its Derivatives in the Biology and Cell Fate Specification of Neural Stem Cells', Mol Neurobiol, 55(9), pp. 7107-7117.

Coronel, R., Palmer, C., Bernabeu-Zornoza, A., Monteagudo, M., Rosca, A., Zambrano, A. and Liste, I. (2019) 'Physiological effects of amyloid precursor protein and its derivatives on neural stem cell biology and signaling pathways involved', Neural Regen Res, 14(10), pp. 1661-1671.

Covic, M., Karaca, E. and Lie, D. C. (2010) 'Epigenetic regulation of neurogenesis in the adult hippocampus', Heredity (Edinb), 105(1), pp. 122-34.

Efthymiou, A., Shaltouki, A., Steiner, J. P., Jha, B., Heman-Ackah, S. M., Swistowski, A., Zeng, X., Rao, M. S. and Malik, N. (2014) 'Functional screening assays with neurons generated from pluripotent stem cell-derived neural stem cells', J Biomol Screen, 19(1), pp. 32-43.

Ertaylan, G., Okawa, S., Schwamborn, J. C. and Del Sol, A. (2014) 'Gene regulatory network analysis reveals differences in site-specific cell fate determination in mammalian brain', Front Cell Neurosci, 8, pp. 437.

Garcia-Osta, A. and Alberini, C. M. (2009) 'Amyloid beta mediates memory formation', Learn Mem, 16(4), pp. 267-72.

Gatt, A., Lee, H., Williams, G., Thuret, S. and Ballard, C. (2019) 'Expression of neurogenic markers in Alzheimer's disease: a systematic review and metatranscriptional analysis', Neurobiol Aging, 76, pp. 166-180.

Gulisano, W., Melone, M., Li Puma, D. D., Tropea, M. R., Palmeri, A., Arancio, O., Grassi, C., Conti, F. and Puzzo, D. (2018) 'The effect of amyloid- $\beta$ peptide on synaptic plasticity and memory is influenced by different isoforms, concentrations, and aggregation status', Neurobiol Aging, 71, pp. 51-60. 
409 Hollands, C., Bartolotti, N. and Lazarov, O. (2016) 'Alzheimer's Disease and Hippocampal Adult 410 Neurogenesis; Exploring Shared Mechanisms', Front Neurosci, 10, pp. 178.

Hsieh, J. (2012) 'Orchestrating transcriptional control of adult neurogenesis', Genes Dev, 26(10), pp. 1010-21.

Kempermann, G., Song, H. and Gage, F. H. (2015) 'Neurogenesis in the Adult Hippocampus', Cold Dale, E., Teich, A. F., Palmeri, A., Ninan, I., Boehm, S. and Arancio, O. (2016) 'Time-dependent reversal of synaptic plasticity induced by physiological concentrations of oligomeric Abeta42: an early index of Alzheimer's disease', Sci Rep, 6, pp. 32553. Regulate Recycling of Synaptic Vesicles via Alpha7 Acetylcholine Receptor and CDK5/Calcineurin Signaling', Front Mol Neurosci, 10, pp. 221.

Li, X., Bao, X. and Wang, R. (2016) 'Neurogenesis-based epigenetic therapeutics for Alzheimer's disease (Review)', Mol Med Rep, 14(2), pp. 1043-53.

Livak, K. J. and Schmittgen, T. D. (2001) 'Analysis of relative gene expression data using real-time quantitative PCR and the 2(-Delta Delta C(T)) Method', Methods, 25(4), pp. 402-8.

Malmsten, L., Vijayaraghavan, S., Hovatta, O., Marutle, A. and Darreh-Shori, T. (2014a) 'Fibrillar betaamyloid 1-42 alters cytokine secretion, cholinergic signalling and neuronal differentiation', J Cell Mol Med, 18(9), pp. 1874-88.

Moreno-Jimenez, E. P., Flor-Garcia, M., Terreros-Roncal, J., Rabano, A., Cafini, F., Pallas-Bazarra, N., healthy subjects and drops sharply in patients with Alzheimer's disease', Nat Med, 25(4), pp. 554-560.

Mu, Y. and Gage, F. H. (2011) 'Adult hippocampal neurogenesis and its role in Alzheimer's disease', Mol Neurodegener, 6, pp. 85.

Pierret, C., Morrison, J. A., Rath, P., Zigler, R. E., Engel, L. A., Fairchild, C. L., Shi, H., Maruniak, J. A. and Kirk, M. D. (2010) 'Developmental cues and persistent neurogenic potential with in an in vitro neural niche', BMC Dev Biol, 10, pp. 5. plasticity and memory', Neurobiol Aging, 33(7), pp. 1484 e15-24. pp. 601.

444 Shohayeb, B., Diab, M., Ahmed, M. and Ng, D. C. H. (2018) 'Factors that influence adult neurogenesis 445 as potential therapy', Trans/ Neurodegener, 7, pp. 4.

446 Tincer, G., Mashkaryan, V., Bhattarai, P. and Kizil, C. (2016) 'Neural stem/progenitor cells in 447 Alzheimer's disease', Yale J Biol Med, 89(1), pp. 23-35. 
448 von Bohlen Und Halbach, O. (2007) 'Immunohistological markers for staging neurogenesis in adult 449 hippocampus', Cell Tissue Res, 329(3), pp. 409-20.

450 Wicklund, L., Leao, R. N., Stromberg, A. M., Mousavi, M., Hovatta, O., Nordberg, A. and Marutle, A. 451 (2010) 'Beta-amyloid 1-42 oligomers impair function of human embryonic stem cell-derived forebrain 452 cholinergic neurons', PLoS One, 5(12), pp. e15600. Lisuk, D., Grasmick, J. M., Silberman, P., Silberman, G., Jappelli, R. and Gage, F. H. (2014) 'Modeling hippocampal neurogenesis using human pluripotent stem cells', Stem Cell Reports, 2(3), pp. 295-310.

Yu, D. X., Marchetto, M. C. and Gage, F. H. (2014) 'How to make a hippocampal dentate gyrus granule neuron', Development, 141(12), pp. 2366-75.

\section{FIGURE LEGENDS}

Figure 1. Phenotypic examination of NPCs directed differentiation in culture. Phase-contrast images at 0, 9, 19 and 29 days of basal cells incubated in expansion medium and control cells incubated in differentiation medium (20 x magnification with $10 \times$ magnification inset, scale bar $100 \mu \mathrm{m})$.

Figure 2. NPCS differentiation immunofluorescence staining. Representative images showing NeuN, GFAP and Synaptophysin protein expression at 9, 19 and 29 days of NPCs incubation in differentiation medium (20 x magnification, scale bar $100 \mu \mathrm{m}$, with $20 \times$ magnification $4 \times 4$ montage inset, scale bar $300 \mu \mathrm{m})$.

Figure 3. Gene expression profiles of analyzed genes NEUROD1 (A), NCAM1 (B), TUBB3 (C), RBFOX3 (D), CALB1 (3) and GFAP (F). Bar graphs show the mRNA percentage of relative expression of each gene relative to the geometric mean of ACTB and GAPDH housekeeping genes expression for basal or control NPCs at each time point of culture. Vertical lines represent the standard error of the mean (SEM). ${ }^{*}$ p-value $<0.05 ;{ }^{*}$ p-value $<0.01 ; * * *$ -

475 value $<0.001 ; * * *$ p-value $<0.0001$

476 Figure 4. $A \beta$ administration effect during the differentiation process time course. mRNA expression of NCAM1 (A), TUBB3 (B) and RBFOX3 (C) genes relative to the geometric mean of $A C T B$ and GAPDH housekeeping genes expression was determined for control and $A \beta$ treated 
479 NPCs. Vertical lines represent the SEM. *p value $<0.05 ;{ }^{* *} p$ value $<0.01 ; * *$ p value $<0.001$;

$480 * * * * \mathrm{p}$ value $<0.0001$

481 Figure 5. Expression pattern of AHN lineage specific genes studied. The diagram illustrates 482 NEUROD1, NCAM1, TUBB3, RBFOX3, CALB1 and GFAP expression profiles during the directed

483 neuronal differentiation of our time window NPCs culture model, according to the 484 developmental stages of AHN within the neurogenic niche of the DG. 


\section{0 days}

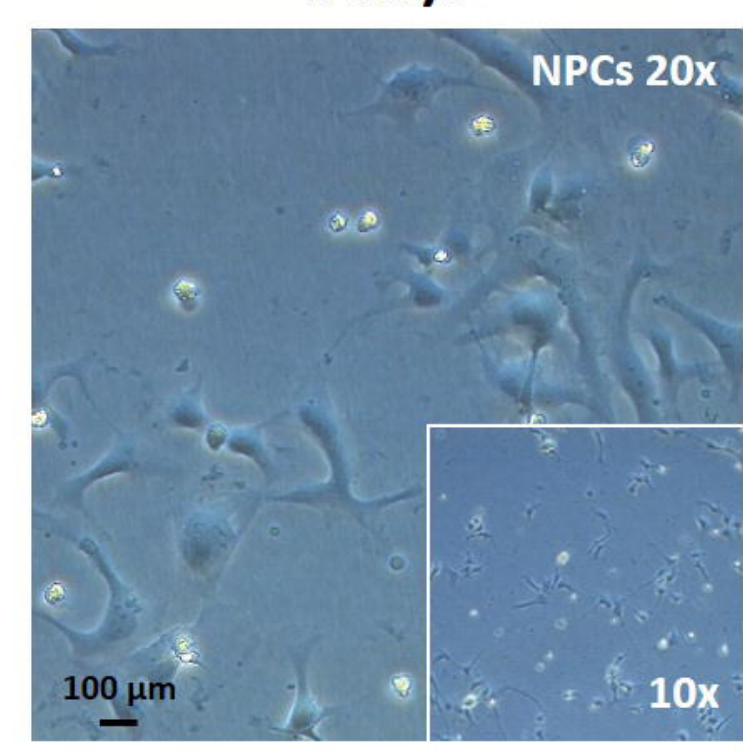

Basal
9 days

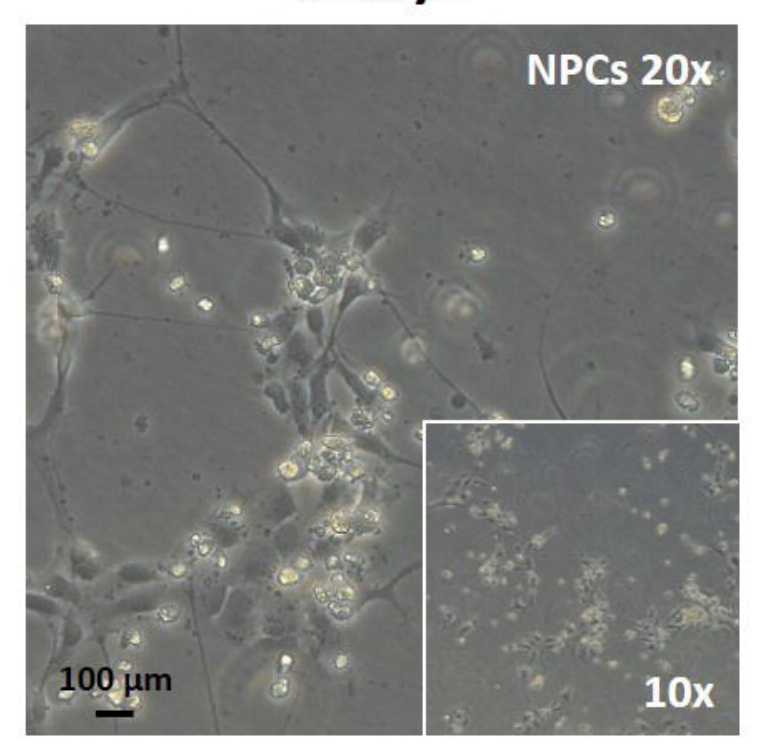

19 days

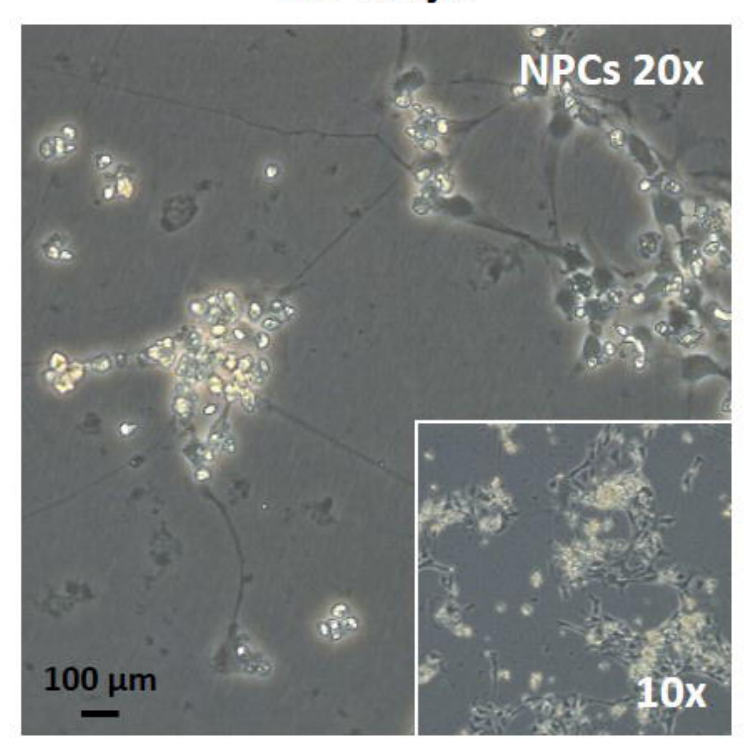

29 days

NPCs 20x

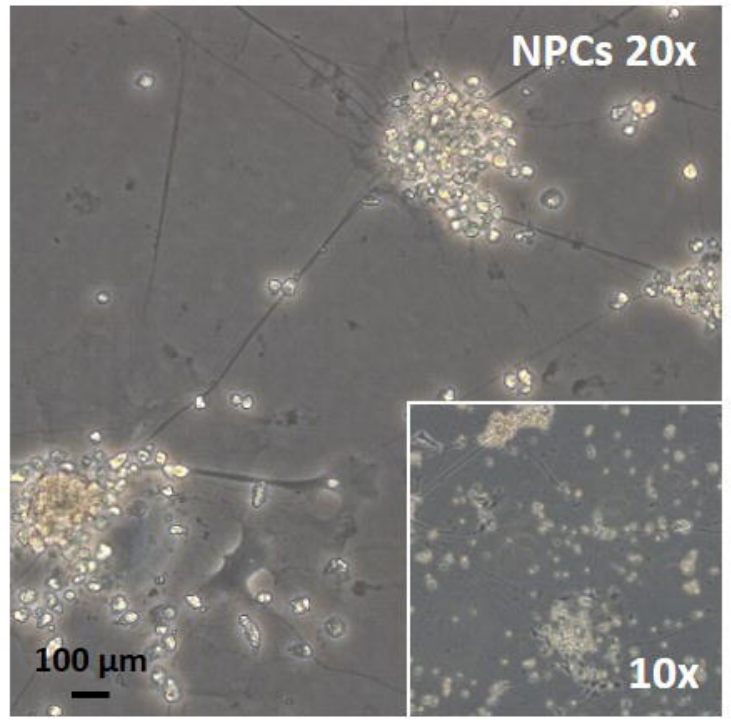


9 days
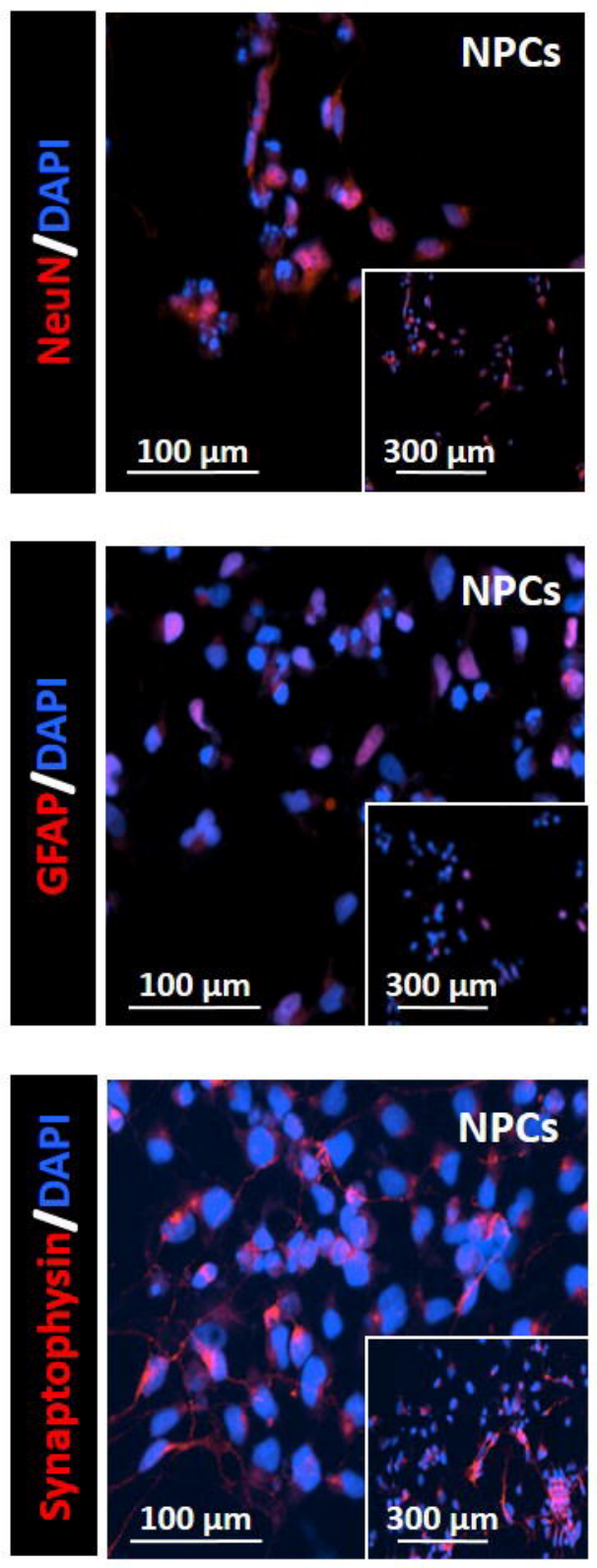

19 days
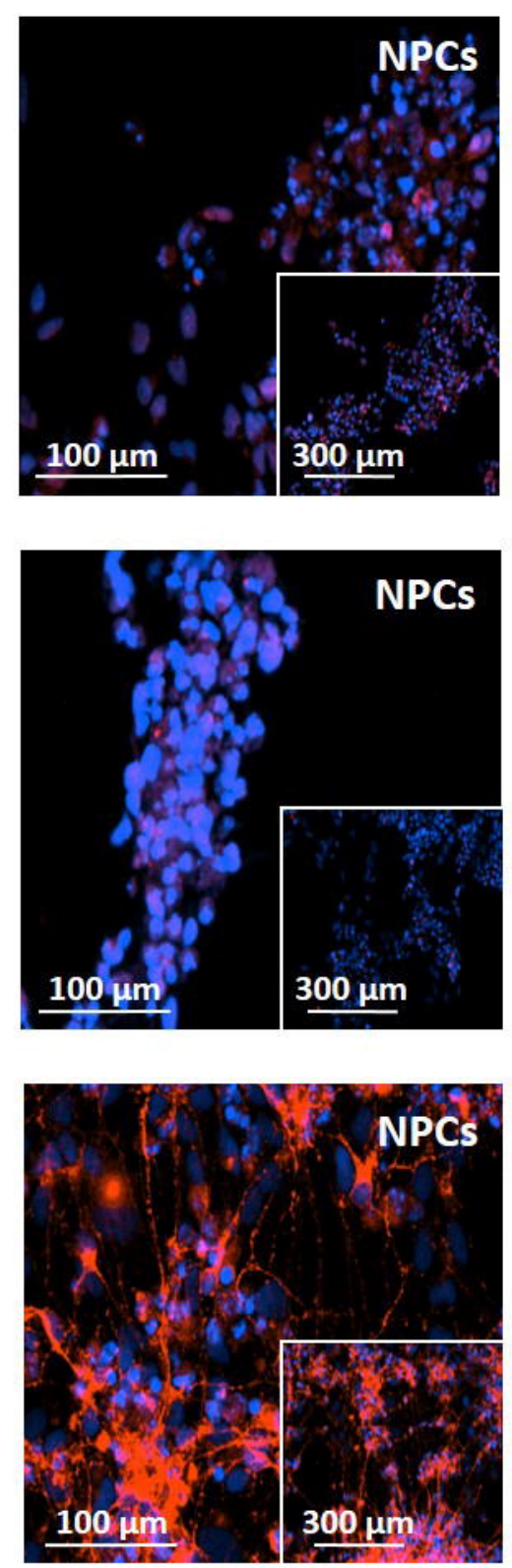

29 days
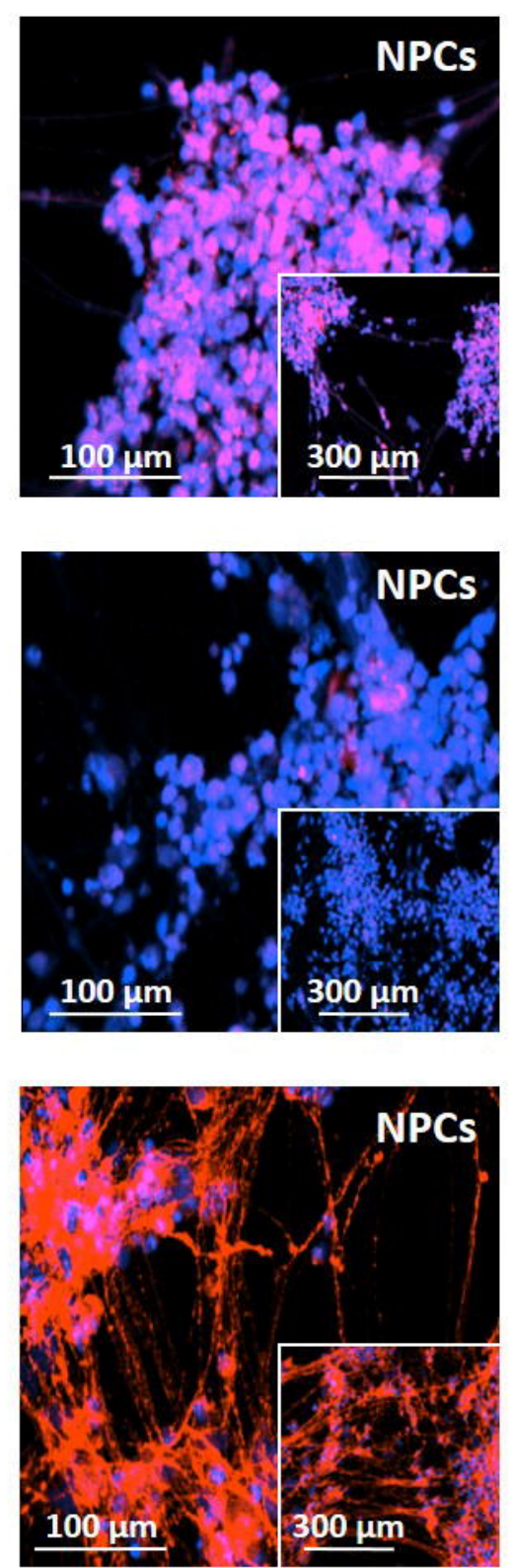
A

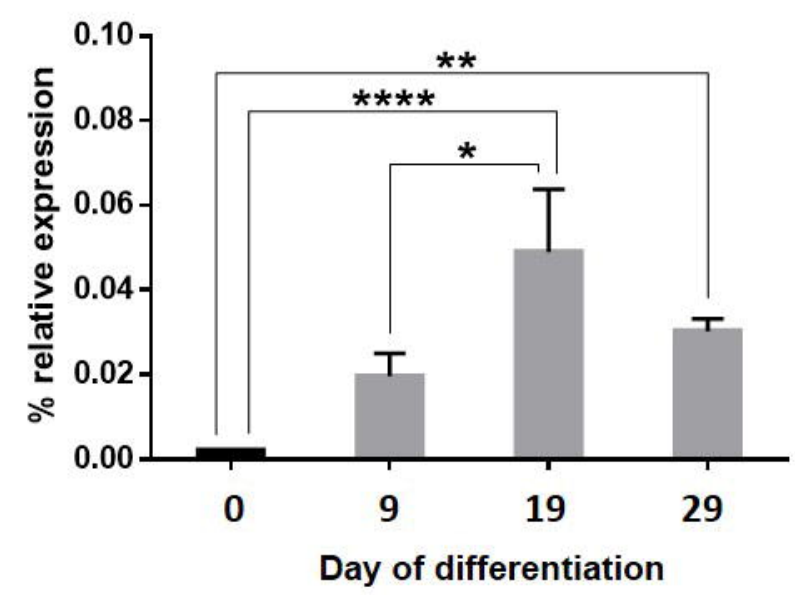

C

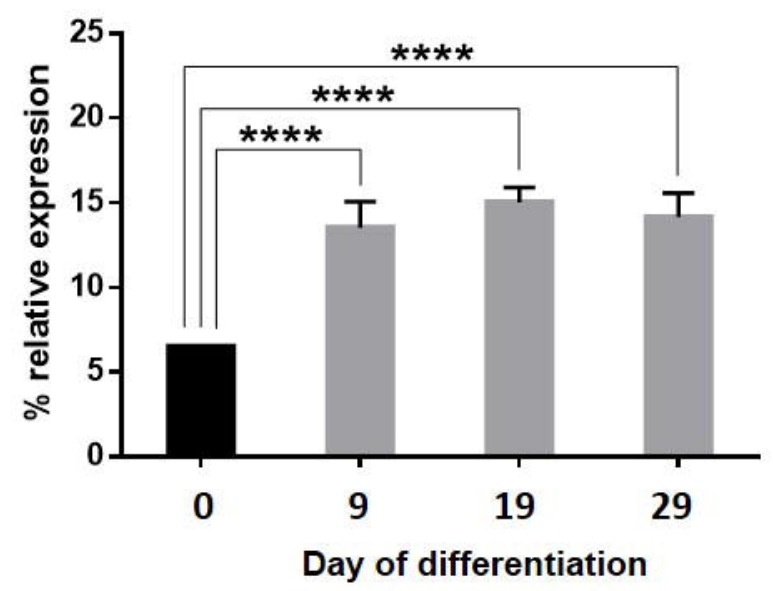

E



B

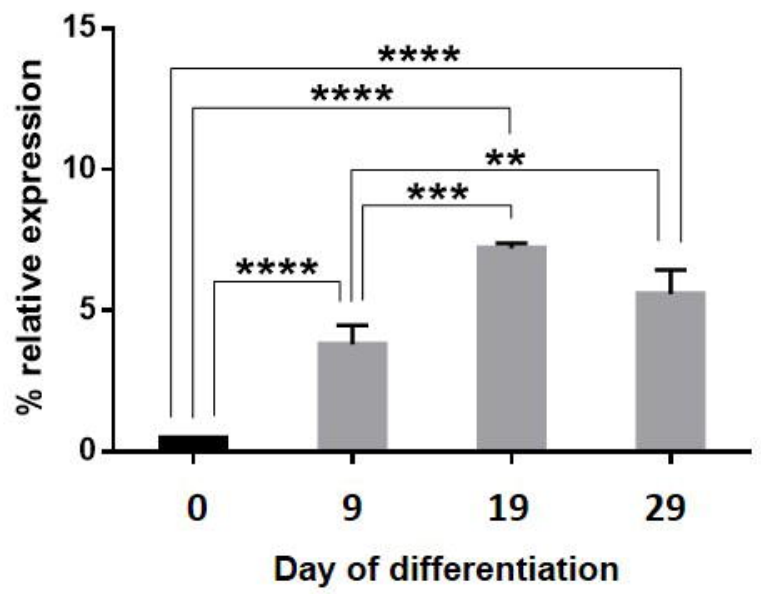

D
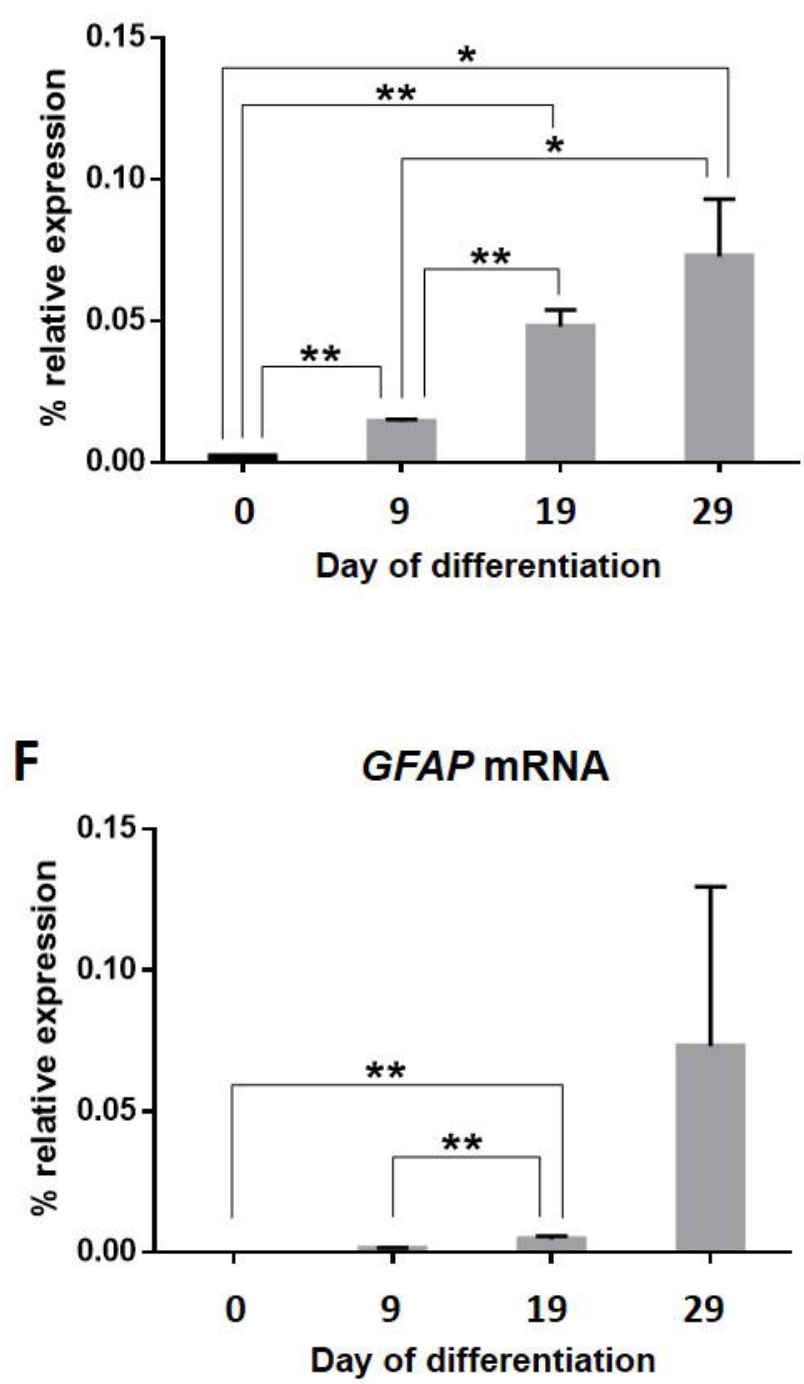
A

NCAM1 mRNA 9 days

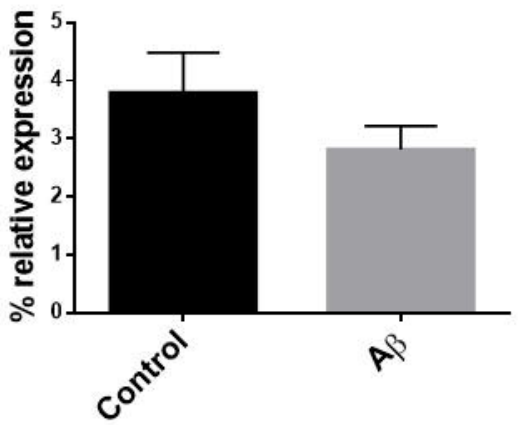

B

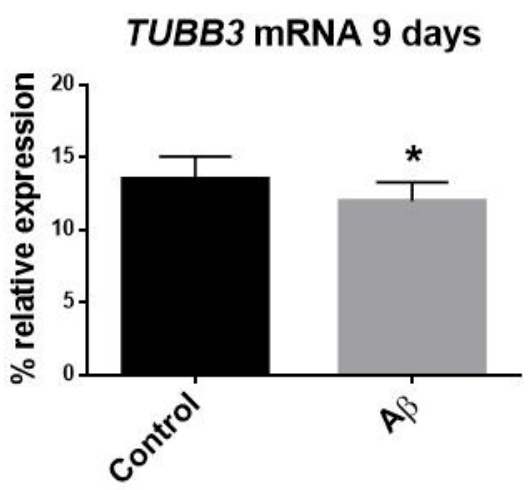

C

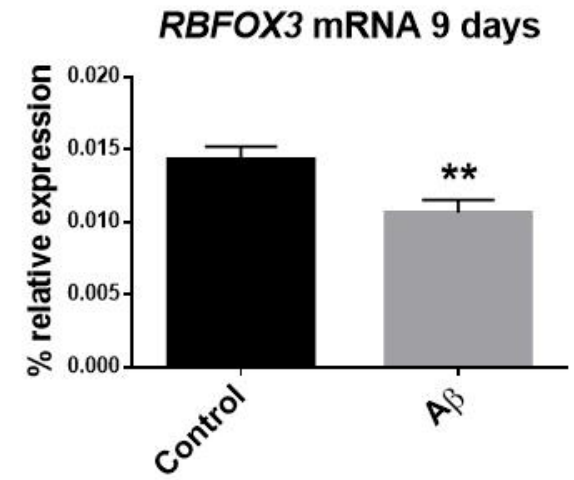

NCAM1 mRNA 19 days

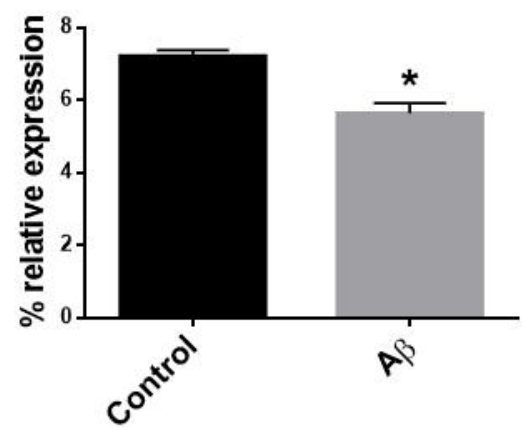

TUBB3 mRNA 19 days

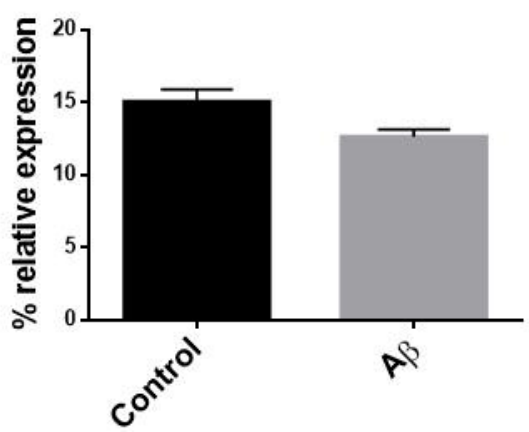

RBFOX3 mRNA 19 days

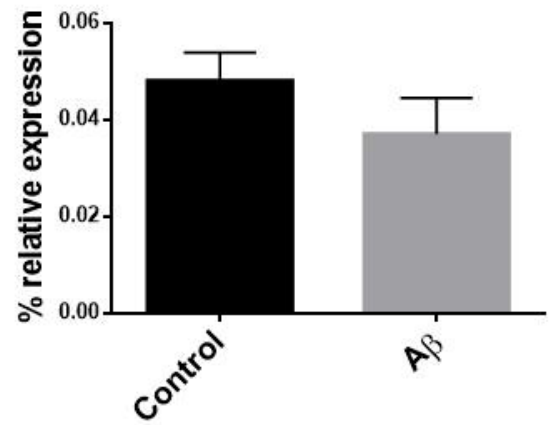

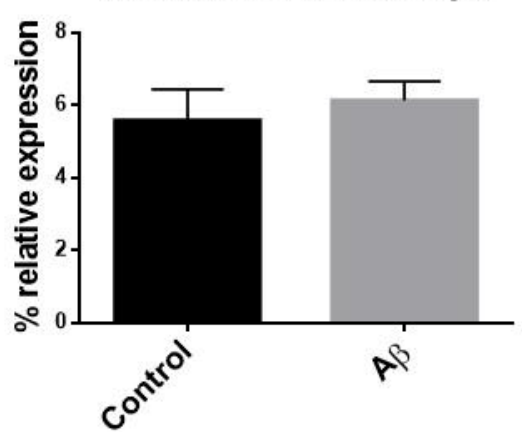

NCAM1 mRNA 29 days

TUBB3 mRNA 29 days

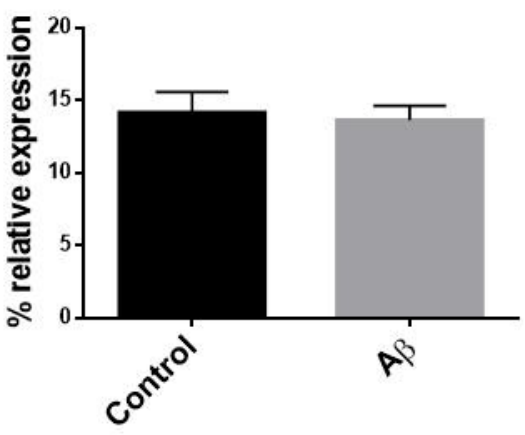

RBFOX3 mRNA 29 days

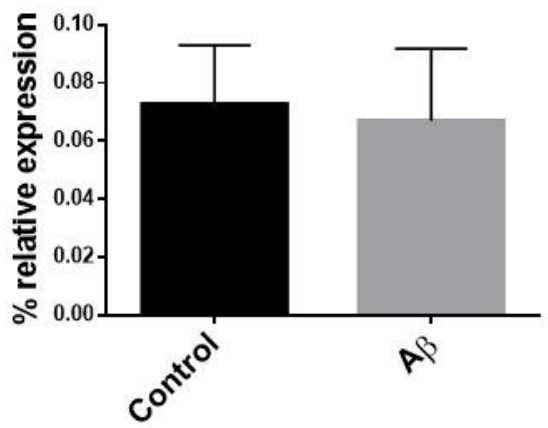




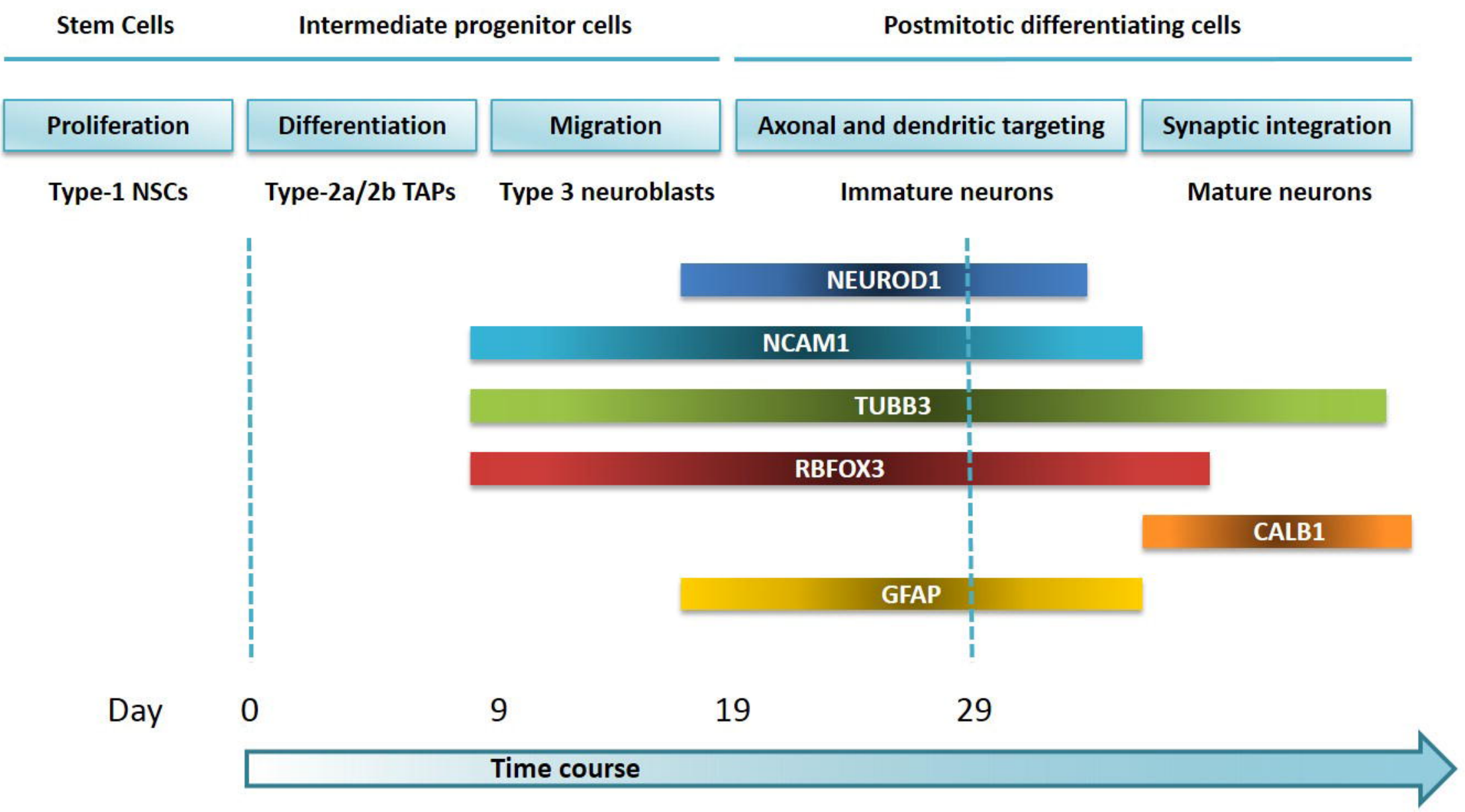

